\title{
CFD Simulations of Flow Around Octagonal Shaped Structures
}

\author{
Benny Gabriel Jebaraj and Freeda Christy C* \\ School of Civil Engineering, Karunya Univeristy. Coimbatore, India
}

Received 28 April 2016; Accepted 1 November 2016

\begin{abstract}
The race for the construction of super-tall structures is the new trend in this era. Construction of super-tall structures is mainly governed by wind and its associated effects on these large slender structures. Hence proper knowledge about the aerodynamic effects of wind and the response of the structure to these effects is highly important. This knowledge helps in arriving at the shape of the building, the structural system to be adopted and also the method of construction to be adopted. Recent advancements in Computational Fluid Dynamics (CFD) helps in predicting the flow of the wind around the structure, the velocity and pressure distributions, lift and drag co-efficient, the formation of vortices and many such parameters that govern the structural stability of the structure. This project studies about the aerodynamic force coefficients on an octagonal building for various angles of attack and are compared with the results obtained from wind tunnel experiments and also studied about the response of the building due to vortex induced forces and moments.
\end{abstract}

Keywords: CFD Simulations, Aerodynamics, Turbulent Flow

\section{Introduction}

Aerodynamics is a branch of dynamics concerned with studying the motion of air, particularly when it interacts with a solid object. Understanding the motion of air around an object (often called a flow field) enables the calculation of forces and moments acting on the object. Wind effects are the challenges that designers have to deal with super-tall building design. In association with high slenderness, low natural frequencies, low inherent damping level and high wind speed at upper lever, super-tall buildings are more susceptible to winds. Modern tall buildings go higher and higher with the advances in structural design and high strength materials. However, every advance in height comes with a new difficulty. Efficient structural systems, high strength materials, and increased height, result with decrease in building weight and damping, and increase in slenderness. Understandably, an appropriate choice of building shape and architectural modifications are also extremely important and effective design approaches to reduce wind induced motion by altering the flow pattern around the building. Along wind or simply wind is the term used to refer to drag forces [1]. Under the action of the wind flow, structures experience aerodynamic forces including the drag (along wind) force acting in the direction of the mean wind flow. The across wind response, is a motion in a plane perpendicular to the direction of wind. In the design of most modern tall buildings, the across wind response often dominates over the along wind response. Cuong Nguyen et al [2] performed a CFD analysis on different aspect ratios of rectangular tall buildings and studied about the dynamic torsional behaviour of tall buildings under wind loads. The numerical results are being comparable with the experimental results. Dong-

*E-mail address: freeda@karunya.edu

ISSN: 1791-2377 @ 2016 Eastern Macedonia and Thrace Institute of Technology. All rights reserved.
Hyeog Yoon et al [3] underwent a parametric study to elucidate the characteristics of flow past a square cylinder inclined with respect to the main flow in the laminar flow regime. Attempt is made to classify the related flow patterns from a topological point of view, resulting in three distinct patterns in total. A comprehensive analysis of the effects of Reynolds number and angle of incidence on flow-induced forces on the square cylinder were studied. Collecting all the results obtained, contour diagrams of force and moment coefficients, Strouhal number, rms of lift-coefficient fluctuation, as well as a flow-pattern diagram are proposed for the ranges of the two parameters considered. Gera et al [4] explored the unsteady flow around a 2D square cylinder using CFD techniques considering laminar flow. The study predicted the influence of Re on quantities such as Strouhal number and lift, drag, and base suction coefficients. In particular, the question of the transitional Reynolds number, i.e. the critical Re above which the flow ceases to be laminar and cannot be made to two dimensional, needs further investigation. Ahmad et al performed a numerical simulation of wind loads on the roofs of several low rise buildings with full scale Reysnold's number, boundary layer and turbulence properties have also been simulated for various angle of wind attacks [5]. The study showed that the numerical simulation of wind loads hold a great potential for extending codes of practice for wind loads. Olawore and Odesola [6] considered the influence of vertical structure and pressure distribution around the section of rectangular cylinders. The Strouhal number, drag coefficient and lift coefficient for various Reynold's number were calculated and thus concluded that vortex shedding phenomenon must be taken into consideration when designing buildings for safe, economical and effective engineering designs. Ankit Mahajan et al [7] made a validation of the CFD model simulation of wind tunnel experiments through the available results experimental work carried out. Three different square 
cylinders were tested with varied angle of incidence from $0^{\circ}$ to $90^{\circ}$ with $15^{\circ}$ variation. After computational study it is was observed that for $0^{\circ}$ wind incidence angle for isolated building model the values of the coefficients from CFD is close to those specified in ASCE 7-02 and AS/NZS 1170.2. On comparison with IS 875 (part 3) it is observed that for front face and side face of the building the values obtained are in close agreement. Kavya et al [8] studied about the 2D unsteady flow past a square cylinder at various angle of incidence and determined that the eddy sizes increases as the angle of incidence increases and both Strouhal number and lift coefficient respond strongly to the orientation of the cylinder. An experimental investigation of surface static pressure distributions on octagonal cylinder in uniform and turbulent flows was carried out on single and a group of cylinders by Md. Jomir Hossain et al [9] for various angle of wind attacks in a wind tunnel and found insignificant results as compared to a sharp - edged square cylinder. This study mainly focusses on flow around an octagonal shaped structure using k-omega SST turbulence model and determine the aerodynamic coefficients and compare them with wind tunnel experiments

Presently this paper focusses on the determination of aerodynamic force and moment coefficients for a building, octagonal in plan using 2D simulations for an angle of attack ranging from $0^{\circ}$ to $50^{\circ}$. The k-omega SST turbulence model is used for the simulations of the flow around the building

\section{Methodology}

The building will be considered as a bluff body and the entire simulations were done using ANSYS FLUENT 15. The building was modelled using ANSYS Design Modeller. The computational domain around the building is considered as shown in Figure 1.

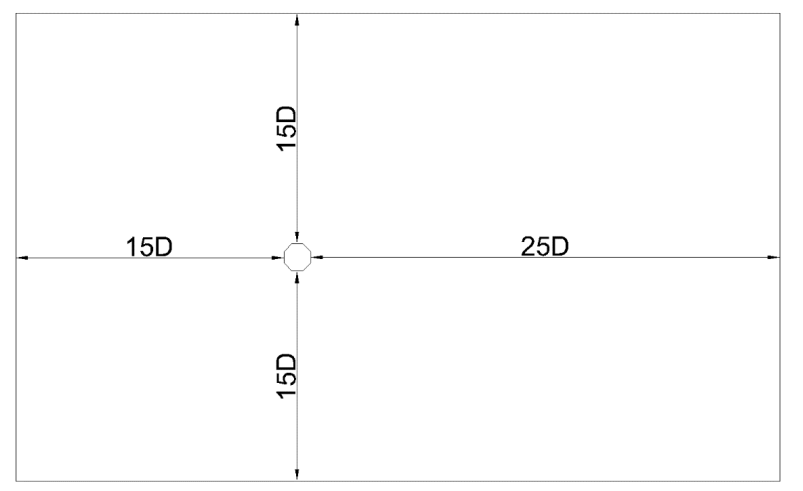

Fig 1.Computational Domain around the building

The building was rotated at an angle of $0^{\circ}$ to $50^{\circ}$ and the domain was modelled. After modelling all the models were imported to ANSYS MESH, a unique software for meshing, and the domain was meshed. The near wall meshing around the building was calculated as given below.

It is given by the equation

$y^{+}=\frac{y u^{*}}{v}$

Where,

$\mathrm{y}^{+}$is a non-dimensional number $\mathrm{u}^{*}$ is shear friction velocity

$\mathrm{y}$ is the distance of the first grid point from the wall $\mathrm{v}$ is the kinematic viscosity

For our problem

Free stream velocity $=13.2 \mathrm{~m} / \mathrm{s}$
Density $=1.225 \mathrm{~kg} / \mathrm{m}^{3}$

Dynamic Viscosity $=1.7894 \mathrm{e}-5 \mathrm{~kg} / \mathrm{ms}$

Boundary layer length $=0.08 \mathrm{~m}$

$\operatorname{Re}=\frac{\rho u D}{\mu}$

$\mathrm{Re}=72000$

Estimated wall distance $=2.0 \mathrm{e}-5 \mathrm{~m}$

Figure 2 shows the meshing around the building and the entire domain for $0^{0}$ angle of attack

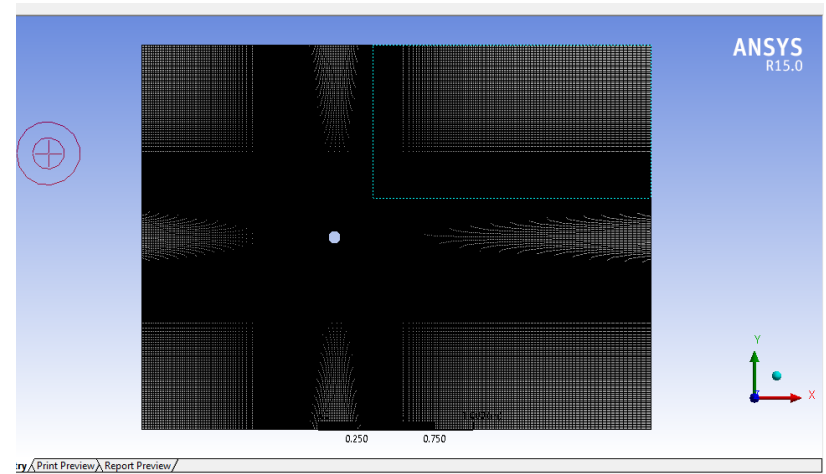

Fig. 2.Computational Domain around the building

Meshing plays a crucial and major role in any CFD project. The accuracy and correctness of any numerical solution depends on the meshing pattern adopted around the building. The first grid was spaced at a distance as calculated from equation 1 . Figure 3 shows the near wall meshing around the building for each angle of attack, from $0^{\circ}$ to $50^{\circ}$ angle of attack.
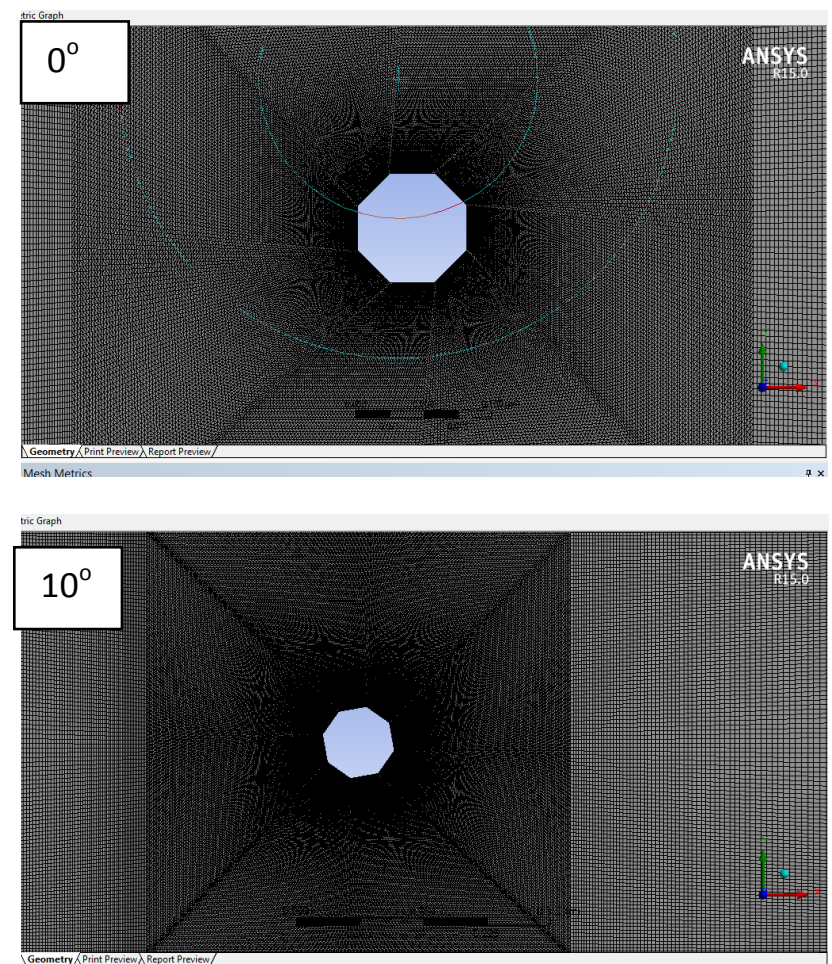

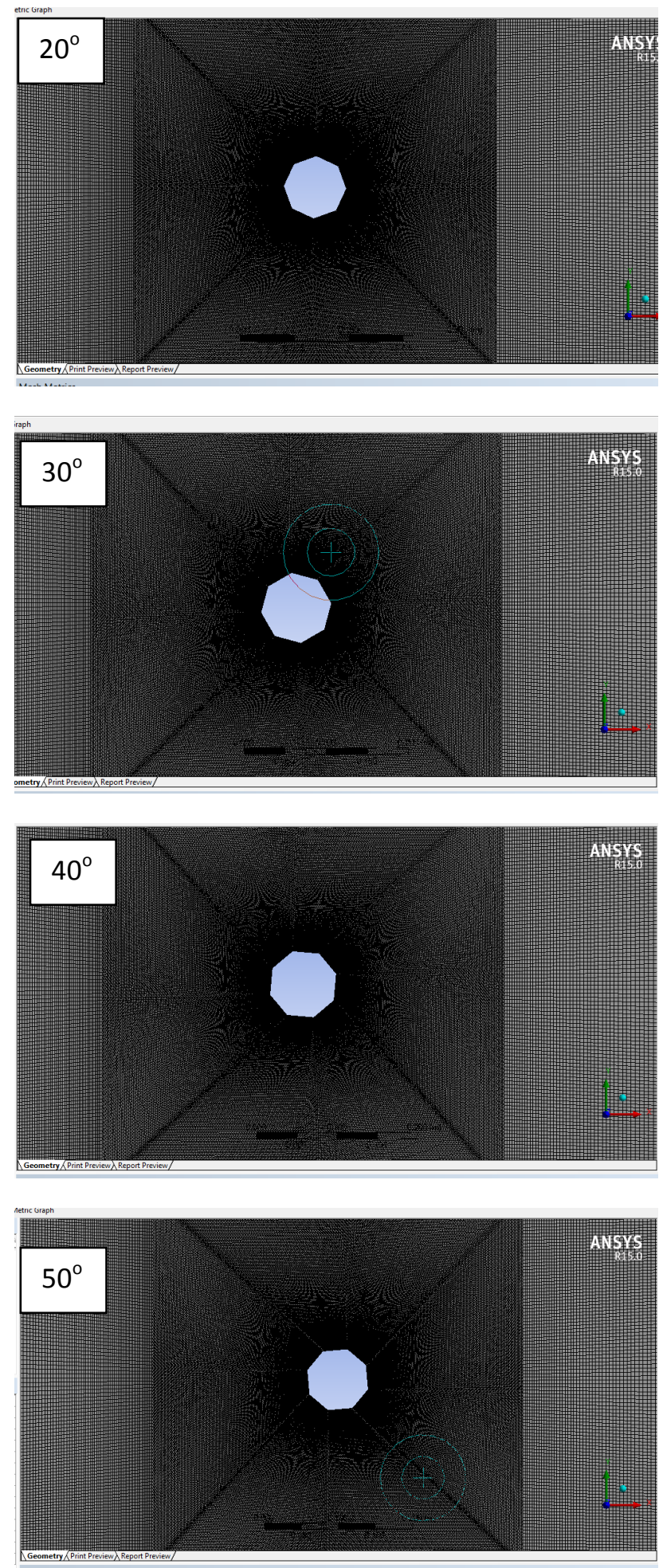

Fig. 3. Near wall meshing around the building

A total number of 254256 elements were generated in each of the models. After the meshing had been successfully completed the mesh models were imported to ANSYS FLUENT.

\section{Fluent Control Parameters}

The following steps were followed for all the models. The imported mesh was checked for any errors and also checked for orthogonality, skewness and also for aspect ratio. The material was selected as air and the default valued of air as given in FLUENT were used.

The boundary condition was set as given below;

Inlet: The inlet condition was set up with a velocity of 13.2 $\mathrm{m} / \mathrm{s}$ in a direction normal to the boundary. Turbulent intensity was given as $0.2 \%$ and Turbulence viscosity ratio was given as 10

Outlet: The gauge pressure was set as $0 \mathrm{~Pa}$. Backflow turbulent parameters were set equal to that of inlet boundary in order to reduce difficulties in convergence

Symmetry: Velocity in normal direction were set to zero along with the derivatives of the flow variables

Wall boundaries: No slip condition was given and velocities in the flow and normal to the flow directions were set to zero

Initial values were set equal to the inlet boundary values

Reference values were also set to compute from the inlet boundary values and the area value was given as the overall depth of the structure

Solution Methods were set to SIMPLE scheme and Second Order Upwind scheme for solving transport equations when transient simulations were performed

Solution Monitors were set to monitor drag, lift and moment coefficient

Initially the calculations were run at steady state simulations for 1200 iterations so that the solution would converge at a faster rate and then continued with transient simulation until the solution converges. Unsteady simulations were carried out at time step of $0.001 \mathrm{~s}$ and maximum iterations per time step were given 75 to monitor lift and drag coefficients until convergence. The drag, lift and moment coefficient values were monitored till the values converge between constant values.

Post-processing of results: The variation of drag coefficient, lift coefficient, moment coefficients were written to a file which will be further used for the interpretation of results using Excel. The plots, contours and velocity vectors were plotted for each of the models for further analysis and comparison of the results.

\section{Results and Discussions}

The models after being analysed were then sent for post processing for viewing the results. The main aim of this project was to study the aerodynamic force and moment coefficients of a building and compare them with wind tunnel analysis. Comparison of drag coefficient, $\left(\mathrm{C}_{\mathrm{d}}\right)$ values for various angles of attack are indicated in Figure 4 . The drag coefficient was an important parameter when it comes to building a tall structure against wind loads. Since the wind being dynamic the flow properties of the fluid are measured with respect to time and the results are plotted. The solution was allowed to converge and the results were plotted.

$$
\mathrm{C}_{\mathrm{d}} \text { vs. angle of attack }
$$

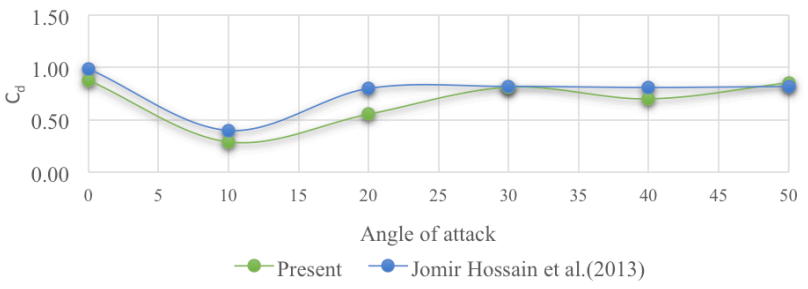

Fig. 4. Drag Coefficient for various angle of attack 
From Figure 4 the variation of the value of $\mathrm{C}_{\mathrm{d}}$ for various angle of attacks seen. On comparing the entire values of $\mathrm{C}_{d}$ over the angle of attack and referring with a work that was already done in wind tunnel experiments, the $C_{d}$ value has a very close correlation with the wind tunnel experiments. There is also a significant drop in the value of $\mathrm{C}_{\mathrm{d}}$ at $10^{\circ}$ angle. This drop was mainly due to the fact that there was minimum area of attack available for the wind to incident on the structure directly and hence there was very little pressure produced in the wake region leading to a drop in the value of $\mathrm{C}_{\mathrm{d}}$.

Comparison of lift coefficient $\left(\mathrm{C}_{1}\right)$ values for various angles of attack are indicated in Figure 5. The coefficient of lift is another important parameter to be considered while designing tall structures. The lift force that occurs on the structure was mainly due to the across-wind motion of the wind. The across-wind motion sets up lateral vibrations in the building.

\section{$C_{1}$ vs. angle of attack}

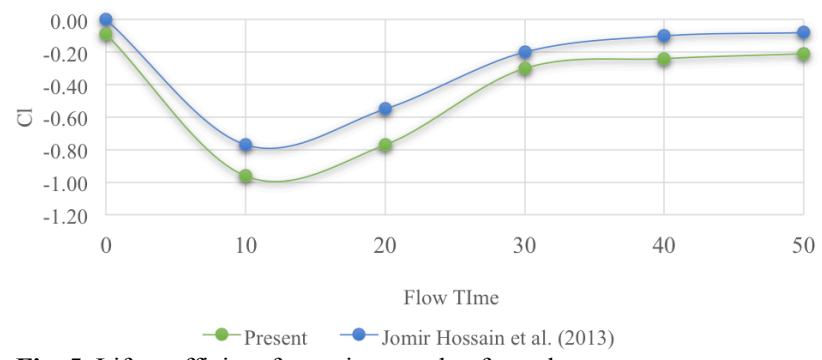

Fig. 5. Lift coefficient for various angle of attack

From Fig 5, the value of the lift coefficient for a time period of $1.2 \mathrm{~s}$ for various angle of attacks are compared. The simulations were stopped since the results started converging. In a similar fashion to the drop in drag coefficient at $10^{\circ}$ there is also a similar drop at an angle of $10^{\circ}$ in the lift coefficient which was due to the uniform distribution of pressure in the wake region. There results obtained were almost similar to the results obtained from wind tunnel experiments.

Comparison of coefficient of moment, $\left(\mathrm{C}_{\mathrm{m}}\right)$ values for various angles of attack were made and indicated in Figure 6. The coefficient of moment values was an important parameter for determining the total moments at the base of the structure. The wind while incident on a structure would produce torsional moments which will produce a twisting action in a direction about the central axis of the structure and along the wind direction.

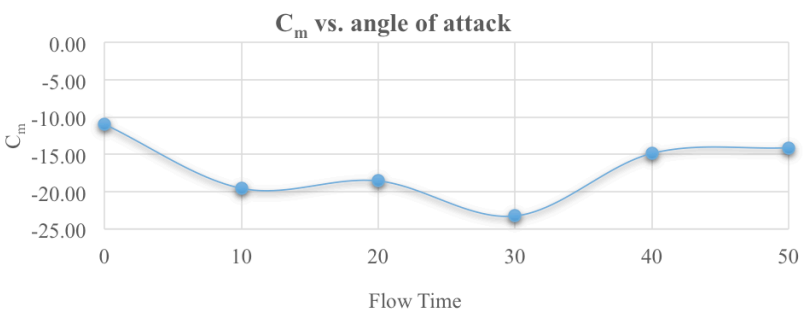

Fig. 6. Moment coefficient for various angle of attack

The values of moment coefficients for various angles of wind attack were shown in Figure 6. There was a maximum moment coefficient for an angle of $30^{\circ}$ of wind angle attack which was the critical value when compared to other angle of wind attacks. This was because at $30^{\circ}$ angle of wind attack, there was more surface area for the wind to incident on the building and this creates more moment.

Contour plot was plotted for pressure distribution on the structure at various angle of attack $0^{\circ}, 10^{\circ}, 20^{\circ}, 30^{\circ}, 40^{\circ}$ and $50^{\circ}$ were indicated in Figure 7, 8, 9, 10, 11 and 12.

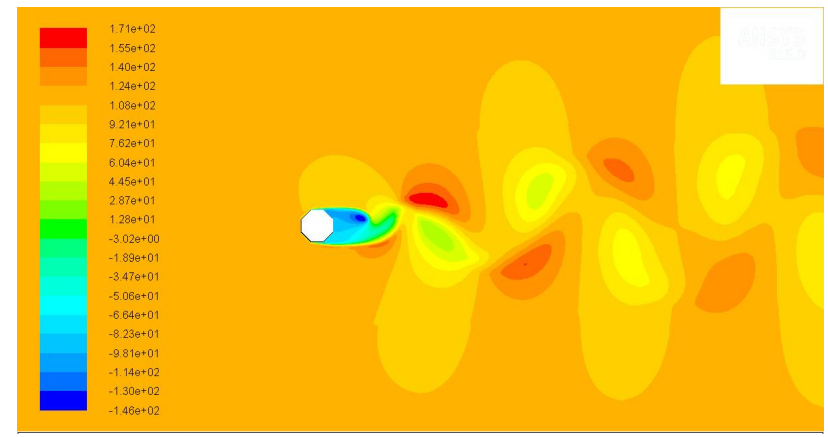

Fig. 7. Pressure Plot for $0^{\circ}$ angle of attack

Figure 7 shows for $0^{\circ}$ angle of attack the negative pressure region was very high near the boundary of the building and dissipates as positive pressure throughout the domain

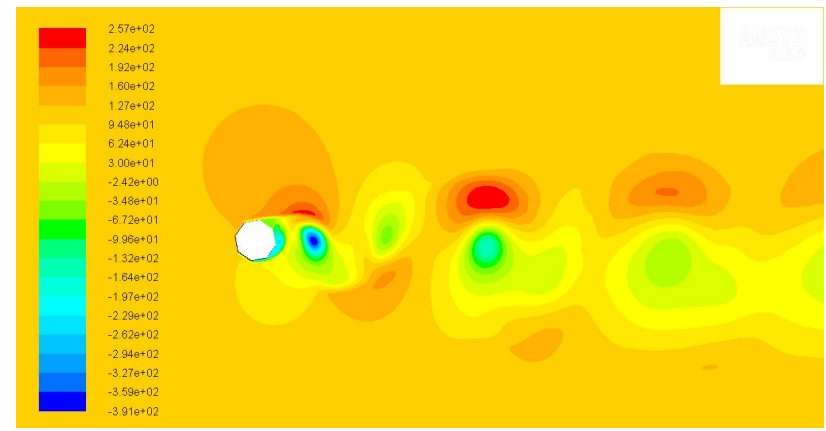

Fig. 8. Pressure Plot for $10^{\circ}$ angle of attack

Figure 8 shows for $10^{\circ}$ angle of attack the negative pressure region had moved away from the building and high pressure regions are formed well away from the building

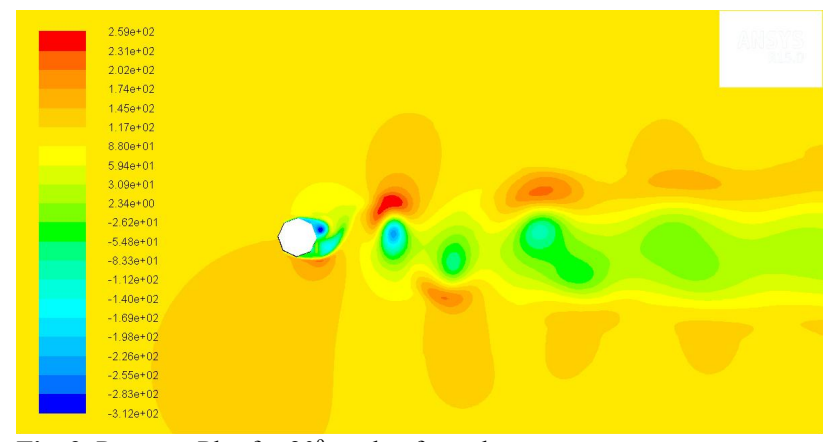

Fig. 9. Pressure Plot for $20^{\circ}$ angle of attack

Figure 9 shows for $20^{\circ}$ angle of attack there was a small very low pressure region near the building and there was well distributed positive pressure regions 


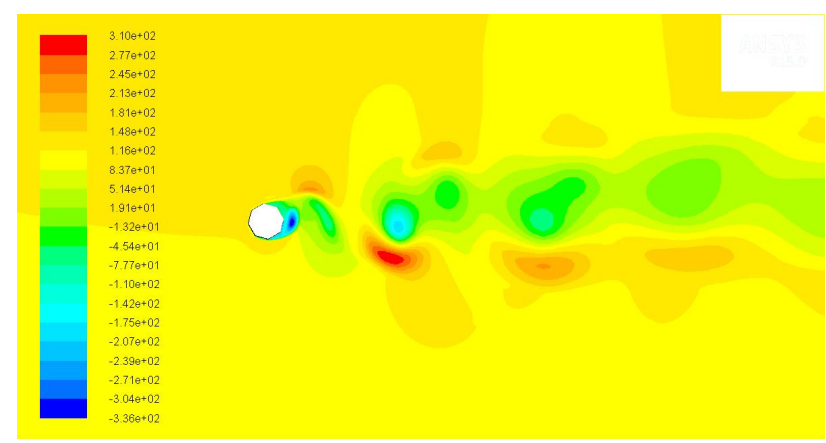

Fig. 10. Pressure Plot for $30^{\circ}$ angle of attack

Figure 10shows for $30^{\circ}$ angle of attack there was also a very low pressure region near the building and the pressure distribution was not much uniform throughout the domain.

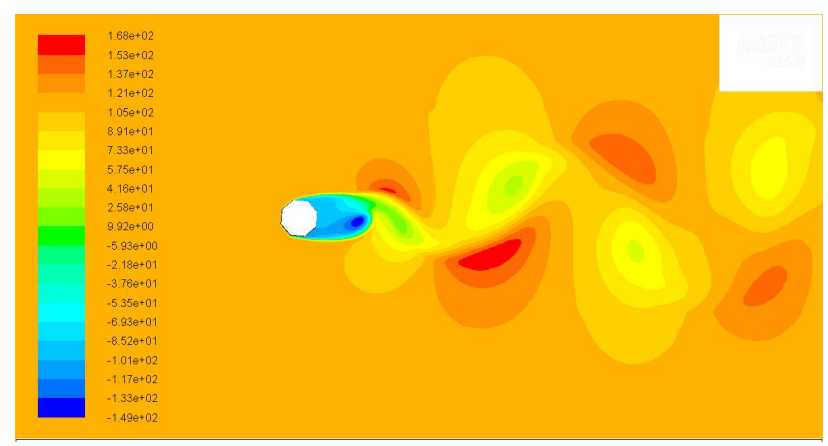

Fig. 11. Total Pressure Plot for $40^{\circ}$ angle of attack

Figure 11 shows for $40^{\circ}$ angle of attack the behaviour of the total pressure distribution around the building was almost similar to that of a circular cylinder

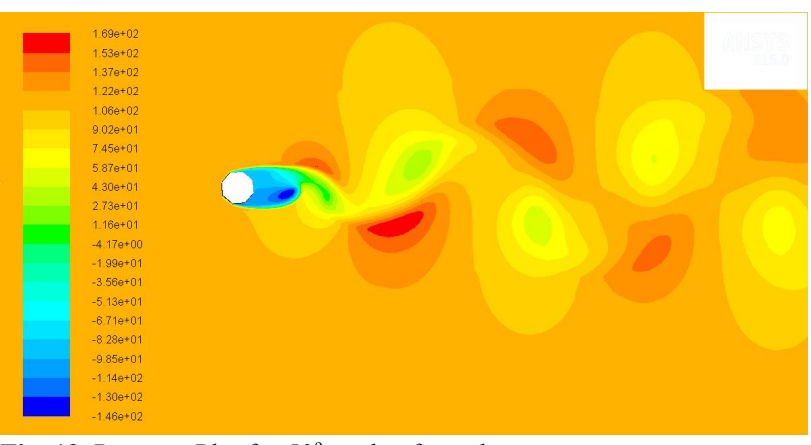

Fig. 12. Pressure Plot for $50^{\circ}$ angle of attack

Figure 12 shows for $50^{\circ}$ angle of attack also the distribution of total pressure is similar to that of a circular cylinder with a very uniform pressure distribution.

\section{Conclusion}

- The k-omega SST turbulence model was well suited for flow around buildings

- The aerodynamic force coefficients obtained for various angles of attack were similar to the results obtained from wind tunnel experiments

- The response of the building due to vortex induced forces and moments was calculated

- Tapered structures perform well in the case of tall buildings subjected to dynamic wind loads

- CFD analysis can be very well adopted for flow around complex shapes and can be a good replacement for wind tunnel experiments

\section{Acknowledgement}

Authors would like to thank Karunya University for providing the facilities to carry out the study.

\section{References}

1. Taranath, B., Structural Analysis, and Design of Tall Buildings, McGraw-Hill Book, 2011

2. Cuong K. Nguyen, Tuan D. Ngo, Priyan A. Mendis, John C.K. Cheung, "Dynamic torsional behavior of tall building under wind loads using CFD approach", The fourth International Symposium on Computational Wind Engineering (CWE2006), Yokohama, 2006, pp $405-408$

3. Dong-Hyeog Yoon, Kyung-Soo Yang and Choon-Bum Choi, "Numerical Study of Flow patterns past an inclined square cylinder", American Institute of Physics, 2010

4. Gera. B, Pavan K, Sharma, Singh R.K, "CFD analysis of 2D unsteady flow around a square cylinder", International Journal of Applied Engineering Research, Vol. 1 Issue 3, 2010, p 602

5. Ahmad. S, Muzzammil. M, Zaheer. I, "Numerical Prediction of wind load on low buildings", International Journal of Engineering, Science and Technology, Vol. 3, No. 5, 2011, pp. 59-72
6. Olawore A.S., Odesola I.F, "2D Flow around a rectangular cylinder: A computational study", International Journal of Science and Technology, Vol 2, No 1, 2013, pp $1-26$

7. Ankit Mahajan, Puneet Sharma, Ismit Pal Singh, "Wind Effects on Isolated Buildings with different sizes through CFD simulation", Journal of Mechanical and Civil Engineering, Volume 11, Issue 3 Ver. IV, 2014, pp 67 - 72

8. Kavya H.P., Banjara Kotresha, KishanNaik, CFD Analysis of 2D unsteady flow past a square cylinder at an angle of incidence, International Journal of Advanced Research in Mechanical and Production Engineering and Development, Vol.1 (2), 2014, pp 117 125

9. Md. Jomir Hossain, Md. Quamrul Islam and Mohammad Ali, "Lift and drag on cylinder of octagonal cross section in a turbulent stream", Journal of Engineering Science \& Technology Review, Vol. 6 Issue 3, 2013, pp 105-110. 